durch den Wechsel der Jahreszeiten, ausgebildet bei jenen Arten und Individuen, welche sich in ihrem Fortpflanzungsgebiete einer periodisch ganz oder teilweise verschwindenden Nahrung angepafst haben. Der Herbstzug ist ein Vorbeugen gegen die durch Nahrungsmangel und Kälte entstehende Herabminderung des Artbestandes, der Frühjahrszug das Aufsuchen bestimmter Fortpflanzungsgebiete, an welche bestimmte Individuen der Art am besten angepafst sind, an welchen dieselben daher zur Fortpflanzung, d. i. zur Erhaltung der Art das Maximum günstiger Lebensbedingungen vorfinden.

Inwieweit sich diese Auffassung des Zuges bewähren wird, möge dahingestellt bleiben. Eine Folge derselben scheint jedoch meiner vielleicht etwas befangenen Meinung nach sicher zu sein, die nämlich, dafs in diesem Sinne geführte Untersuchungen in erster Linie immer nach dem Feststellen von Tatsachen trachten werden, was der Forschung keineswegs zum Schaden gereichen kann.

Ich möchte noch bemerken, dafs ich mit Vorwissen sämtliche spekulative und theoretische Schlufsfolgerungen, selbst die nächstliegenden $\mathrm{zu}$ vermeiden suchte und in diesem Vortrage nur das aussprechen wollte, was auf Tatsachen gegründet werden konnte.

Bevor ich schliefse, möchte ich als dankbarer Schüler nicht versäumen, ausdrücklich zu betonen, dafs diese Zugsstudie zugleich eine Demonstration der Methode der U. O. C. ist, welche Methode von meinem hochverehrten Chef und Lehrmeister Otto Herman begründet wurde.

\title{
Über die Vogelwelt der Halbinsel Hela.
}

Vortrag, gehalten auf der Jahresversammlung der D. O. G. in Danzig am 3. Oktober 1908 von Th. Zimmermann.

\section{Hochgeehrte Versammlung!}

Auf Anregung des Leiters der Vogelwarte Rossitten, des Herrn Dr. Thien emann, habe ich mich seit dem Jahre 1906 zur Zeit des Vogelzuges im Frühjahr und Herbst einige Wochen auf der Halbinsel Hela zwecks ornithologischer Beobachtungen aufgehalten. Die Halbinsel Hela erstreckt sich, wie sie auf der ausgestellten Karte sehen, von Nordwest nach Südost in die Ostsee, in einer Länge von $35 \mathrm{~km}$. Das Fischerdorf Hela, welches sich allmählich zu einem vielbesuchten Badeorte mit grofsartigem Kurhause, mit Hôtels, Villen etc. vermausert hat und von den Bewohnern Danzigs vielfach besucht wird, liegt auf der südwestlichen Spitze der Halbinsel. Hinter dem $13 \mathrm{~km}$ langen Kiefernwalde, - von dem Dorfe Hela gerechnet, - zeigt das Gelände dünenartigen Charakter, welches rechts von einem magern, bald breiteren, bald schmaleren Kiefernwalde eingesäumt ist. An der 
Küste der Putziger Wieck, 13 klmtr vom Dorfe Hela entfernt, liegen die Ortschaften Danziger Heisternest, 1 klmtr weiter Putzig, von Heisternest $5 \mathrm{klm}$ weiter Kufsfeld und $8 \mathrm{klmtr}$ weiter Ceynova. Von dort nach dem ersten Dorfe auf dem Festlande - Grofsendorf - beträgt die Entfernung noch weitere $8 \mathrm{klmtr}$. Ich will noch bemerken, dafs die Breite der Halbinsel bei dem Dorfe Hela am gröfsten ist, fast 4 klmtr, weiter hinauf wechselt die Breite aufserordentlich, bei Kufsfeld beträgt sie nur $1 / 2$ klmtr. Wenn nun die Halbinsel geographisch auch nicht so ganz in der Zugrichtung der Vögel liegt, so glaubten wir doch - Freund Thienemann und ich, - dafs diese Landzunge den im Herbste von NO über die See kommenden Vögeln und den im Frühjahr von SW. Heraufwandernden vielleicht als günstiger Anflugs resp. Rastpunkt dienen könnte. Jedenfalls wollte ich gleich damals im Frühjahr einen Versuch machen und zusehen, ob sich im Frühjahr Vogelzug auf der Halbinsel bemerkbar mache. Ich begab mich Mitte April 1906 zum ersten Male nach Hela und im Verlaufe der Wochen weiter die Halbinsel hinauf. Ich will gleich bemerken, dafs ich zu ganz überraschenden Resultaten kam. Einen ersten ausführlichen Bericht über den Vogelzug auf Hela 1906 erstattete ich im hiesigen botan. zoolog. Vereine im Febr. 1907; derselbe ist im Jahresbericht des Vereins enthalten. Dort habe ich weitere Details betreffs der über die Halbinsel wandernden Vogelarten, speziell über den grofsartigen Raubvogelzug dort gegeben, über die Eigenart der polnisch-katholischen Bewohner der Fischerdörfer, über die fast völlige Unfruchtbarkeit des Bodens, - zumeist Sand mit spärlichem Graswuchs - kein Halm Getreide wird auf der Halbinsel gebaut, über Fischerei u. s. w. Es würde zu viel Zeit beanspruchen, wollte ich mich ausführlich darüber äufsern; ich verweise auf den Jahresbericht des Vereins. Einen zweiten Bericht habe ich im Verein im Februar d. J. gegeben, die Beobachtungen aus diesem Jahre ruhen vorläufig noch in meinem Tagebuche. Einen Auszug aus dem Tagebuche erhält Freund Thienemann alljährlich nach Schlufs der Zugperiode; er äufserte wiederholt, dafs ihm die Zugbeobachtungen von Hela wertvoll seien. - Ein längerer Aufenthalt auf der Halbinsel in den sehr primitiven Gasthäusern der armseligen Fischerdörfer ist durchaus nicht als reizvoll $\mathrm{zu}$ bezeichnen; die Verpflegung - in der Hautsache Fische - allerdings in erfreulicher Abwechslung, meistens aber Flundern in Margarine gebacken, - zu Mittag und abends - läfst viel zu wünschen übrig. Das nächtliche Lager ist auf Seegras, das Deckbett wiegt durchschnittlich $25 \mathrm{klgr}$ ! Doch ein eifriger Ornithologe, der hungrig und müde von den längeren Beobachtungstouren und der Jagd heimkommt, und der dann noch bis in die späte Nacht am Arbeitstische sitzt, um die Tagesbeute zu präparieren, macht sich aus dieser Lebensmisere nichts, er ist froh und guter Laune, denn draufsen im krüppligen Kiefernwalde, auf den mageren Wiesen mit ihren Tümpeln, auf 
der grofsen See und am Wieckstrande entwickelt sich während der Zugzeiten ein herzerfreuendes Vogelleben. Die sichtbaren Resultate meines Aufenthaltes auf der Halbinsel während der Zugzeiten der 3 letzten Jahre in Gestalt von Vogelbälgen habe ich dort ausgelegt; ich bitte die sich für jene Präparate interessirenden Anwesenden, sich nach Schlufs der Sitzung nach vorne zu bemühen behufs genauerer Besichtigung.

Ich habe noch ein paar Bälge von in Westpreufsen seltneren Vogelarten der Sammlung dort beigefügt und zwar einen Balg der Gebirgsbachstelze Motacilla sulphurea Naum.; diese Art brütet alljährlich in einigen Paaren an den Schneidemühlen im Schmelztale bei Sagorsch. Ferner Bälge vom Zwergfliegenfänger; dieser Vogel ist in Westpreulsen stellenweise recht häufig, was Herr Amtsrichter Dr. Henrici bereits vor Jahren constatiert hat. Das Vögelchen wird leicht übersehen, da es sich meistens hoch in den dicht belaubten Baumkronen der Rotbuchen aufhält. Der dort aufgestellte Seestrandläufer Tringa maritima (Brünn.) ist ein recht seltener Gast an der Ostseeküste, er ist auf der sogen. Messinainsel bei östlich Neufähr erlegt und im Besitz des Herrn Glaubitz - Langfuhr - eines eifrigen Ornithologen. Ich habe den Herren immer um den Vogel beneidet, nun kann ich diese tadelnswerte Gefühlsregung ablegen: ich hatte vorgestern die Freude, in den Besitz eines solchen Vogels zu kommen und zwar von der Halbinsel aus Ceynova; das Balgpräparat befindet sich ebenfalls dort in der Sammlung. Den Balg eines weifssternigen Blaukehlchen besitze ich leider noch immer nicht, obwohl das Vögelchen nach Angabe des hier anwesenden Herrn Professor Ibart dicht an den Mauern hiesiger Stadt, aber auf gesperrtem Gelände alljährlich brütet. Auf Hela wird diese Art regelmäfsig anfangs April beobachtet. Zwei Bälge des für Preufsen ziemlich seltenen Mornellregenpfeifer befinden sich noch dort, sie gehören meinem Freunde Ulmer - Quanditten - , der die beiden Vögel auf seinem Vorwerke Taplacken in Ostpreufsen am 26. August d. J. erlegt hat, er schickte sie mir zur Präparation.

\section{Ektoparasiten der Vögel.}

\section{Bericht}

\section{von Dr. P. Speiser (Sierakowitz)}

über seinen am 3. Oktober in Danzig gehaltenen Vortrag.

Zur gesamten Naturgeschichte eines Tieres gehört allemal auch die Erkenntnis der Beziehungen zu anderen Tieren, sei es als erbeutender Räuber, sei es als Beute oder als Wirt für Parasiten. Und das genauere Studium dieser Beziehungen kann manchesmal Verhältnisse aufdecken, die für das Verständnis der übrigen Biologie des Tieres von wesentlicher Bedeutung sind. 


\section{$2 \mathrm{BHL}$ Biodiversity Heritage Library}

1909. "Über die Vogelwelt der Halbinsel Hela." Journal fu

r Ornithologie 57, 98-100. https://doi.org/10.1007/bf02094652.

View This Item Online: $\underline{\text { https://www.biodiversitylibrary.org/item/43500 }}$

DOI: https://doi.org/10.1007/bf02094652

Permalink: https://www.biodiversitylibrary.org/partpdf/142391

\section{Holding Institution}

MBLWHOI Library

\section{Sponsored by}

MBLWHOI Library

\section{Copyright \& Reuse}

Copyright Status: No known copyright restrictions as determined by scanning institution.

This document was created from content at the Biodiversity Heritage Library, the world's largest open access digital library for biodiversity literature and archives. Visit BHL at https://www.biodiversitylibrary.org. 